Revue

Revue de l'histoire des religions

de Ihistoire des religions

\title{
Angelo Di BERARDINo (dir.), avec la collab. de Thomas
} C. ODEN, Joel C. ELOWSKY et James HoOVER, Encyclopedia of Ancient Christianity

3 vol., Downers Grove, IVP Academic (InterVarsity Press), 2014

Dominic Moreau

\section{(2) OpenEdition}

Journals

Édition électronique

URL : http://journals.openedition.org/rhr/10978

DOI : 10.4000/rhr.10978

ISSN : 2105-2573

Éditeur

Armand Colin

Édition imprimée

Date de publication : 1 mars 2021

Pagination : 125-128

ISBN : $978-2-200-93375-3$

ISSN : 0035-1423

Référence électronique

Dominic Moreau, «Angelo Dı Berardino (dir.), avec la collab. de Thomas C. Oden, Joel C. Elowsky et James Hoover, Encyclopedia of Ancient Christianity », Revue de l'histoire des religions [En ligne], 1 | 2021 mis en ligne le 19 mars 2021, consulté le 31 mars 2021. URL : http://journals.openedition.org/rhr/ 10978 ; DOI : https://doi.org/10.4000/rhr.10978

Ce document a été généré automatiquement le 31 mars 2021.

Tous droits réservés 


\title{
Angelo Di BERARDINo (dir.), avec la collab. de Thomas C. ODEN, Joel C. ELOWSKY et James Hoover, Encyclopedia of Ancient Christianity
}

\author{
3 vol., Downers Grove, IVP Academic (InterVarsity Press), 2014
}

\section{Dominic Moreau}

\section{RÉFÉRENCE}

Angelo Di BERARDINo (dir.), avec la collab. de Thomas C. ODEN, Joel C. ELOWSKY et James HOOVER, Encyclopedia of Ancient Christianity [=EAC], 3 vol., Downers Grove, IVP Academic (InterVarsity Press), 2014, XXXVIII-937 - XXXIV-1020 - XXXIV-994 p., 26,7 cm, 450 \$, ISBN 978-0-8308-2943-9 (3 vol.).

Entre 1983 et 1988, l'Istituto Patristico Augustinianum faisait paraître, sous la direction d'A. Di Berardino, les trois volumes (1:A-F;2:G-Z; $3:$ Atlante patristico, indici) de la première édition de son Dizionario patristico e di Antichità cristiane $=$ DPAC (Casale Monferrato/Gênes, Marietti). Sans remplacer totalement les très grands dictionnaires encyclopédiques antérieurs (en premier lieu le Dictionnaire d'archéologie chrétienne et de liturgie, mais aussi le Dictionnaire de théologie catholique, le Dictionnaire de droit canon et le Dictionnaire de la Bible de Fulcran Vigouroux) ou toujours en cours (le Dictionnaire d'histoire et de géographie ecclésiastiques par exemple) - le DPAC est d'ampleur plus humble -, l'ouvrage s'est tout de même rapidement imposé comme l'usuel le plus pratique pour les spécialistes $\mathrm{du}$ domaine. Depuis, aucune des entreprises "concurrentes " n'a réussi à vraiment le dépasser; on pense ici, avant tout, à l'Encyclopedia of Early Christianity dirigé par Everett Ferguson, avec la collaboration de

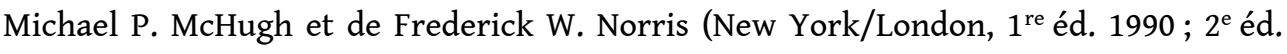
1997). 
2 Le succès et la valeur du DPAC ont incité à sa traduction en dehors de l'Italie. Une version française, ne reprenant que quelques éléments du troisième volume (dont la version originale comprenait des tables chronologiques, un atlas, l'iconographie illustrant les articles et un index analytique), fut ainsi publiée en 1990, sous la direction de François Vial, avec pour titre de Dictionnaire encyclopédique du christianisme ancien (Paris, Cerf). L'ouvrage parut sept ans après l'original, sans aucune addition ni mise à jour, ce qui attira rapidement les critiques (voir, par exemple, Ysabel de Andia dans les Archives de Sciences sociales des religions, 76, 1991, p. 252).

3 Les responsables des traductions espagnole (Diccionario patrístico y de la Antigüedad cristiana, trad. Alfonso Ortiz García et José Manuel Guirau, Salamanque, Ediciones Sígueme ["Verdad e imagen», 97 et 98], 1991-1992; réimpr. 1998) et anglaise (Encyclopedia of the Early Church, trad. Adrian Walford, Cambridge/New York, 1992) ne firent pas exactement les mêmes choix éditoriaux. Dans ces deux versions, la bibliographie a été mise à jour. La valeur du complément bibliographique offert par William H. C. Frend à l'édition anglaise fut, d'ailleurs, si unanimement reconnue par la communauté scientifique internationale, qu'il permit de l'élever au rang d'édition de référence de l'ouvrage (EAC, I, p.IX). Cette qualité, due également au fait que la traduction anglaise est la seule à retenir l'essentiel du troisième volume du DPAC original, lui fut reconnue jusqu'à la publication d'une nouvelle édition italienne dans la deuxième moitié des années 2000.

De 2006 à 2010 parurent ainsi progressivement les quatre volumes (1:A-E ; 2 : F-O ; 3 : $\mathrm{P}-\mathrm{Z} ; 4$ : Tavole cronologiche e sinottiche, indice analitico, carta delle principali sedi episcopali nel Tardo Antico) du Nuovo dizionario patristico e di Antichità cristiane = NDPAC (Gênes/Milan, Marietti 1820), toujours sous le patronage de l'" Augustinianum » et sous la direction d'Angelo Di Berardino. Cette nouvelle édition, plus ample que la précédente, fait une place beaucoup plus importante aux traditions d'Europe orientale et du Proche-Orient, tout en proposant, d'après la préface, une « révision de toutes les entrées, avec correction et mise à jour du texte et de la bibliographie, effectuée, dans la mesure du possible, par les auteurs originaux " (EAC, I, p. X). Plusieurs articles ont été entièrement réécrits et de nombreuses révisions des données archéologiques ont été faites, alors qu'autour de 500 nouvelles notices ont été ajoutées. Parmi ces nouveaux articles, un certain nombre est consacré à différents textes apocryphes ainsi qu'à des évêques de Rome absents de la première édition.

5 Pour cette toute nouvelle édition, le plan du volume complémentaire du DPAC original n'a pas été repris à l'identique. Le nombre pages en témoigne: VII-417 pages pour le tome de 1988 contre XI-144 pages pour celui de 2010. C'est que les parties «iconographie " et "atlas» n'ont pas été retenues, pour être publiées indépendamment (A. Di Berardino [dir.], avec la collab. de Gianluca Pilara, Atlante storico del cristianesimo antico, Bologne, EDB, 2010 ; trad. angl. : Historical Atlas of Ancient Christianity, St. Davids [PA], ICCS Press, 2013). N'ont donc été conservés, pour le quatrième volume du NDPAC, que les tables chronologiques et l'index analytique, auxquels a toutefois été ajoutée une carte topographique dépliable, dite des "principaux sièges épiscopaux dans l'Antiquité tardive » (remarquons que la version anglaise de cette carte a été publiée par ICCS Press, de manière indépendante, en format dépliable ou en format affiche).

6 Ce quatrième volume est un élément qui n'a pas été repris dans la dernière version en date de l'ouvrage, soit la traduction anglaise augmentée de 2014, qui constitue 
désormais le dictionnaire encyclopédique de référence dans le domaine de l'étude du christianisme ancien. D'emblée, on ne peut ainsi que regretter que si peu de bibliothèques ouest-européennes possèdent l'ouvrage ici recensé (d'après le SUDOC, seulement trois bibliothèques universitaires en France) et c'est justement là l'une des raisons principales qui justifie la présente recension, six ans après sa parution. À la décharge desdites bibliothèques, on peut toutefois déplorer que les responsables $d u$ dictionnaire aient entrepris une mise à jour à peine quatre ans après la publication du dernier volume du NDPAC, sachant que l'ouvrage complet valait plusieurs centaines d'euros (440 d'après le site Internet de l'éditeur).

Quoi qu'il en soit, l'EAC présente le plan suivant :

-Vol. 1 - p. [I]-xxxvIII : considérations initiales, dont la préface à la deuxième version anglaise par T. C. Oden (p.IX), traduction anglaise de la préface de 2006 à la deuxième édition italienne d'A. Di Berardino (p.x), traduction anglaise de la préface de 1983 à la première édition italienne aussi par A. Di Berardino (p. XI-xI), notes sur la présentation de l'ouvrage (p.xIII), liste des contributeurs (p.xIV-XVIII), abréviations bibliques (p.xIX) et abréviations utilisées (p. XX-XXXvIII) ; p. 1-912 : articles de A à E; p. 913-937 : liste complète des articles des trois volumes.

- Vol. 2 / Vol. 3 - p. [I]-XxxIv : considérations initiales, dont des notes sur la présentation de l'ouvrage (p.xx), liste des contributeurs (p.x-xiv), abréviations bibliques (p.xv) et abréviations utilisées (p. XVI-Xxxıv) ; p. 1-995: articles de F à O / p. 1-969: articles de P à Z; p. 996-1020 / p. 970-994 : liste complète des articles des trois volumes.

L'éditeur a donc fait le choix de reprendre systématiquement les outils permettant de lire l'ouvrage (abréviations, etc.) en tête de chacun des volumes, de même que la liste complète des articles, à la fin de ceux-ci, ce qui est plutôt pratique, car cela évite le passage d'un tome à l'autre pendant la lecture. Néanmoins, il est dommage que l'élément le plus fondamental du quatrième volume du NDPAC n'ait pas été retenu, à savoir l'index analytique. Attendu qu'il était déjà en grande partie établi, il aurait suffi de le reprendre et d'y ajouter des éléments extraits des nouveaux articles ajoutés. Ces nouveautés sont au nombre de 30 , ce qui élèverait maintenant à 3220 le nombre d'articles, écrits par 266 contributeurs, provenant de 26 pays différents (vol. I, p. IX) : amour, bien, Capoue, Carmen de synodo Ticinensi, Chine, cosmopolitisme, diakonia / diaconat, Dialogi de sancta Trinitate V-VI, dynamis/energeia, éternité, Hiérotheos, incubatio, infini/infinitude, libelli miraculorum, liberté/libre arbitre, Mara bar Sérapion (lettre de), mort, oikeiôsis, pardon, portier, présanctifié, Sérapéion (Sérapéum), siège apostolique, sous-diacre, Théosébie, Triumphus Christi heroicus, Tychon, unité, vieillesse, et Virgo Parens. Par ailleurs, l'article « matristique » a été mis à jour.

Il serait évidemment impossible ici de revenir sur l'ensemble des très nombreuses entrées qui composent l'EAC. Évidemment, l'entreprise est gigantesque et chaque article est le fruit de choix opérés, pour pouvoir définir de manière à la fois succincte et précise, à l'intention d'un public scientifique et estudiantin, des concepts, des personnages et des faits historiques et théologiques. Un certain nombre d'éléments posent tout de même plus de difficultés que d'autres, pour la plupart liés à la prétendue mise à jour globale annoncée par les directeurs de publication, non seulement dans la préface du NDPAC (EAC, I, p.x), mais aussi dans celle de la traduction anglaise ici recensée (I, p. IX). 
10 Pour ne donner que quelques exemples, la notice consacrée à Denys le Petit (I, p. 715) passe sous silence le rôle politico-religieux qu'il a pu jouer, dans le parti laurentien, pendant le schisme romain opposant Laurent à Symmaque, comme si son œuvre canonique avait été simplement celle d'un érudit totalement détaché du monde. Pourtant Eckhard Wirbelauer, dans son Zwei Päpste in Rome. Der Konflikt zwischen Laurentius und Symmachus (498-514). Studien und Texte, Munich, 1993, p. 134-138, a bien démontré qu'il n'en est rien. Cet ouvrage, qui est le plus important sur ledit schisme, est, d'ailleurs, absent aussi de la bibliographie associée à la notice sur Laurent (II, p. 529; l'ouvrage apparaît toutefois sous l'article sur Symmaque - III, p. 667), un personnage qualifié d'antipape dans le dictionnaire. Cette prise de position est problématique du point de vue de l'historien, du fait que Laurent a été considéré, du moins pendant un certain temps, comme un évêque légitime de Rome, par une grande partie de ses contemporains, voire de la tradition postérieure (comme en témoigne, notamment, sa présence dans l'antique série des portraits papaux de la basilique SaintPaul-hors-les-Murs ; voir Luciano De Bruyne, L'antica serie di ritratti papali della basilica di S. Paolo fuori le Mura, Rome, Pontificio Istituto di archeologia cristiana ["Studi di Antichità cristiana », 7], 1934, p.176, fig. 64). - Autre, et dernier, exemple de bibliographie qui aurait demandé à être mise à jour, la référence la plus récente sous la notice « Patrimoine de St.-Pierre » (III, p. 90) remonte à 1997. La lecture d'un article de l'auteur des présentes lignes aurait pourtant permis d'aller plus loin pour la partie antique : «Les patrimoines de l'Église romaine jusqu'à la mort de Grégoire le Grand. Dépouillement et réflexions préliminaires à une étude sur le rôle temporel des évêques de Rome durant l'Antiquité la plus tardive ", Antiquité tardive, 14, 2006, p. 79-93.

11 Certes, il s'agit là de points mineurs qui n'enlèvent rien à l'importance de l'ouvrage, au regard de la masse d'information qu'il livre. Malgré les quelques critiques qui ont été ici exposées, témoignant surtout du fait que la dernière traduction en date du NDPAC n'a pas fait l'objet d'une mise à jour aussi exhaustive qu'annoncée dans sa préface - et on comprend aisément l'ampleur et la difficulté de la tâche -, l'EAC demeure, à ce jour, le dictionnaire encyclopédique sur le christianisme ancien de référence. Par comparaison, le Brill Encyclopedia of Early Christianity ( https://brill.com/view/db/eeco? rskey=UbHzaQ\&result=1), dont la finalisation est prévue pour 2022 et pour lequel A. Di Berardino est consultant, compte cumuler autour de 1300 articles, soit moins de la moitié de ceux contenus dans l'ouvrage ici recensé.

\section{AUTEURS}

\section{DOMINIC MOREAU}

Université de Lille /

UMR 8164-HALMA, Villeneuve d'Ascq. 